\title{
Utilização do método FDTD na modelagem de linhas telefônicas
}

\author{
Rafael C. Oliveira , Aldebaro B. R. Klautau Jr`, Licinius D. S. Alcantara
}

\begin{abstract}
Resumo-Neste artigo, o método numérico das Diferenças Finitas no Domínio do Tempo (FDTD) é aplicado na simulação da transmissão de sinais banda-larga ao longo de linhas telefônicas. $O$ aproveitamento da base de linhas telefônicas por parte de alguns serviços, visando o acesso do usuário final à Internet, justifica o interesse nesta ferramenta de simulação.
\end{abstract}

Palavras-Chave - linhas de transmissão, eletromagnetismo aplicado, Linha Digital do Assinante.

Abstract - In this paper, the Finite-Difference Time-Domain numerical method (FDTD) is applied on the simulation of broadband signal transmission over telephone lines. The use of telephone lines by some services, for last mile Internet access, justifies the interest in this simulation tool.

Keywords - transmission lines, applied electromagnetism, Digital Subscriber Line.

\section{INTRODUÇÃO}

Devido ao advento da Internet, linhas telefônicas são vistas como uma alternativa econômica para a transmissão de dados de modo que a rede mundial de computadores se torne acessível ao usuário final a um custo relativamente baixo. Sistemas xDSL aproveitam a estrutura já existente de parestrançados para tornar a Internet acessível ao usuário final.

A qualificação de enlaces de pares trançados para serviços xDSL consiste em verificar a sua potencialidade de transmitir sinais cujos espectros vão relativamente bem além da faixa espectral de voz, utilizada pelo serviço de telefonia convencional (POTS). Esta qualificação possibilita avaliar a condição da linha para a transmissão de dados a altas taxas de bits com a finalidade de prover uma conexão de internet confiável e rápida. Procedimentos e cenários para testes de linhas telefônicas destinadas a serviços xDSL estão padronizados em [1].

Algumas descontinuidades, tais como cargas indutivas (load-coils) e ramificações (bridged-taps), podem surgir ao longo da linha telefônica e comprometer a transmissão em freqüências mais elevadas, precisando ser localizadas e removidas usando técnicas como Reflectometria no Domínio do Tempo (TDR), por exemplo.

O método numérico da Diferenças Finitas no Domínio do Tempo (FDTD) foi proposto por Yee [2] originalmente para a simulação de ondas eletromagnéticas. $O$ método pode ser

\footnotetext{
- Universidade Federal do Pará, Departmento de Engenharia Elétrica e de Computação, Laboratório de Processamento de Sinais, Belém, PA, Brazil. Email: licinius@ufpa.br
}

empregado no modelo circuital de uma linha de transmissão, pois neste modelo, tensão e corrente podem ser vistas como uma onda TEM (Transversal Eletrica e Magnética) propagando na linha. Este trabalho tem como objetivo utilizar o método FDTD na simulação sinais transmitidos em linhas telefônicas.

\section{O MÉTODO FDTD REVISADO}

Um sinal qualquer que propaga em uma linha de transmissão é descrito pelas equações abaixo [3].

$$
\begin{aligned}
& -\frac{\partial V(z, t)}{\partial z}=R(z) I(z, t)+L(z) \frac{\partial I(z, t)}{\partial t}, \\
& -\frac{\partial I(z, t)}{\partial z}=G(z) V(z, t)+C(z) \frac{\partial V(z, t)}{\partial t},
\end{aligned}
$$

onde $V$ e $I$ são os valores de tensão e corrente do sinal, respectivamente; $R, L, C$ e $G$ são a resistência, a indutância, a capacitância e a condutância distribuída da linha, respectivamente. Por meio destas fórmulas, o sinal pode ser monitorado em um dado instante de tempo $(t)$ em qualquer posição da extensão da linha (z).

O método FDTD consiste na discretização das equações acima, por meio da substituição das derivadas parciais por diferenças finitas, considerando que os valores discretos de tensão e corrente são posicionados de forma intercalada entre si, tanto no tempo como no espaço. Após a discretização, as equações (1) e (2) assumem a forma abaixo.

$$
\begin{aligned}
V_{i}^{n+1} & =\frac{\left(C_{i}-\frac{G_{i} \Delta t}{2}\right) V_{i}^{n}-\frac{\Delta t}{\Delta z}\left(I_{i+\frac{1}{2}}^{n+\frac{1}{2}}-I_{i-\frac{1}{2}}^{n+\frac{1}{2}}\right)}{C_{i}+\frac{G_{i} \Delta t}{2}}, \\
I_{i+\frac{1}{2}}^{n+\frac{1}{2}} & =\frac{\left(L_{i+\frac{1}{2}}-\frac{R_{i+\frac{1}{2}} \Delta t}{2}\right) I_{i+\frac{1}{2}}^{n-\frac{1}{2}}-\frac{\Delta t}{\Delta z}\left(V_{i+1}^{n}-V_{i}^{n}\right)}{L_{i+\frac{1}{2}}+\frac{R_{i+\frac{1}{2}} \Delta t}{2}},
\end{aligned}
$$

onde $\Delta t$ é o passo de tempo, $\Delta z$ é o passo longitudinal, $n$ é o índice temporal e $i$ é o índice longitudinal. No modelo, é considerada a possibilidade dos valores dos elementos distribuídos, $R, L, C$ e $G$, variarem ao longo do eixo da linha. 


\section{SIMULAÇÃO DE UM ENLACE PONTO-A-PONTO}

Um programa FDTD, baseado na metodologia exposta, foi escrito em MatLab. Para validar o algoritmo, foi adotada a linha de transmissão ilustrada na Fig. 1.

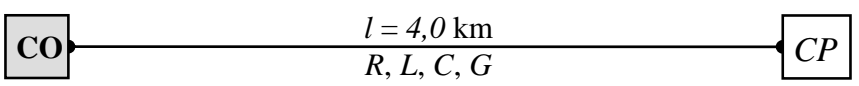

Fig. 1. O enlace telefônico adotado para fins de simulação, o qual conecta o escritório central (central office - CO) a um assinante (costumer premises $\mathrm{CP})$.

Uma linha de extensão de 4,0 km foi considerada. Os passos longitudinais e de tempo adotados são $\Delta z=5 \mathrm{~m}$ e $\Delta t=15 \mathrm{~ns}$, respectivamente. Os valores dos componentes distribuídos são $\mathrm{R}=488.857 \times 10^{-3} \Omega / \mathrm{m}, \mathrm{L}=586.966 \mathrm{nH} / \mathrm{m}, \mathrm{C}=50 \mathrm{pF} / \mathrm{m}$ e $\mathrm{G}=0$.

A Fig. 1 mostra a evolução de um pulso ao longo da linha, o qual foi tomado em três instantes de tempo distintos. Este pulso foi excitado em $\mathrm{z}=0$ e apresenta as característitcas de ser gaussiano no tempo com largura espectral unilateral (3dB) de $1 \mathrm{MHz}$. Pode-se observar a atenuação sofrida pelo pulso na linha, à medida em que propaga.

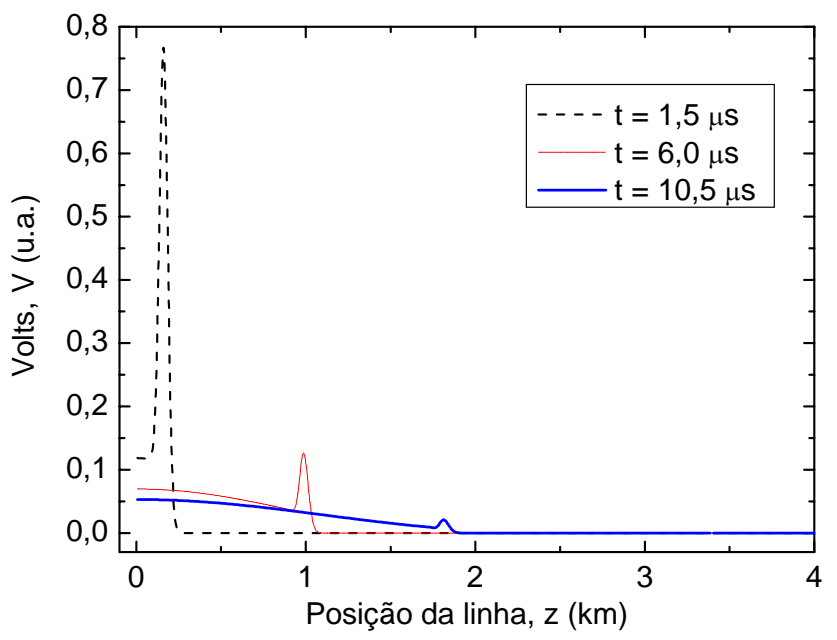

Fig. 2. Evolução de um pulso gaussiano no tempo ao longo da linha, tomado em três instantes de tempo distintos.

A partir da Fig. 2, pode-se estimar a velocidade de propagação do pulso. Considerando as posições do pulso em $t=1,5 \mu$ s e em $t=10,5 \mu$ s, tem-se que

$$
v_{P(F D T D)} \approx \frac{1815 \mathrm{~m}-160 \mathrm{~m}}{10,5 \mu \mathrm{s}-1,5 \mu \mathrm{s}} \approx 1,839 \times 10^{8} \mathrm{~m} / \mathrm{s}
$$

enquanto que a velocidade teórica de propagação do pulso é de $v_{P}=(\sqrt{L C})^{-1}=1,846 \times 10^{8} \mathrm{~m} / \mathrm{s}$. Isto significa um desvio relativo de $0,38 \%$ entre o resultado numérico e teórico.

Com relação à atenuação, considerando uma dependência $V=V_{0} \exp (-\alpha d)$, onde $\alpha$ é a constante de atenuação, o valor medido pela Fig. 2 foi de $\alpha_{\text {FDTD }} \approx 0,0021789$, enquanto que o valor teórico é dado por $\alpha=\mathfrak{R} e\{\sqrt{(R+j 2 \pi f L)(G+j 2 \pi f C)}\}$, $\alpha=0,0022367$, para uma frequência de $f=0,5 \mathrm{MHz}$, a qual está no centro da largura espectral do pulso de excitação.

A Fig. 3 mostra a função de transferência obtida através da relação das DFTs (Transformadas Discretas de Fourier) dos registros temporais da tensão, na entrada e na saída da linha de transmissão, ou seja, $V_{\text {out }}(f) / V_{\text {in }}(f)$. O gráfico foi obtido numa única simulação a partir da utilização de um pulso de excitação gaussiano no tempo, desta vez com largura espectral unilateral (3dB) de $5 \mathrm{MHz}$.

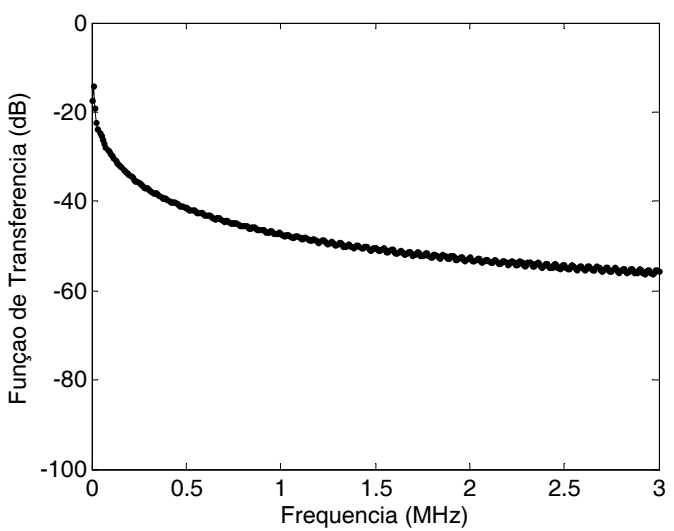

Fig. 3. Função de transferência versus frequência obtido através do FDTD para a linha telefônica em análise, considerando um comprimento de $4 \mathrm{~km}$.

\section{CONCLUSÃO}

Este trabalho ilustra a aplicação do método FDTD nas equações diferenciais de linhas telefônicas. Os resultados são coerentes e a metodologia pode ser aprimorada visando a caracterização de topologias de linhas mais complexas.

\section{AGRADECIMENTOS}

Os autores agradecem ao Programa de Bolsas de Iniciação Científica da UFPA, ao CNPq, ao Laboratório de Processamento de Sinais (LAPS/UFPA) e à Ericsson do Brasil.

\section{REFERÊNCIAS}

[1] ITU-T Recommendation G.996.1, “Transmission Systems and Media - Test Procedures for Digital Subscriber Line (DSL) Transceivers”, 1999.

[2] K. S. Yee, "Numerical solution of initial boundary value problems involving Maxwell's equations in isotropic media," IEEE Trans. Antennas Propagat., vol. 14, pp. 302-307, May 1966.

[3] T. Starr, J. M. Cioffi, and P. J. Silverman, Eds., Understanding Digital Subscriber Line Technology. New York: Prentice Hall, 1999. 